\title{
Publisher Correction to: Existence and a general decay result for a plate equation with non-linear damping and a logarithmic source term
}

\author{
Mohammad M. Al-Gharabli And Salim A. Messaoudi
}

Publisher Correction to: J. Evol. Equ. 18 (2018), 105-125 https://doi.org/10.1007/s00028-017-0392-4

Due to an error in the typesetting process, reference [1] is incorrectly published in the original publication of the article. It should be replaced by the following one:

Alabau-Boussouira F., Convexity and weighted integral inequalities for energy decay rates of nonlinear dissipative hyperbolic systems, Appl. Math. Opt. 51(1), 61-105 (2005).

See also the following reference [2] in the Journal of Evolution Equations where similar techniques and results are discussed.

Alabau-Boussouira F., Piecewise multiplier method and nonlinear integral inequalities for Petrowsky equation with nonlinear dissipation, J. Evol. Equ. 6, 95-112 (2006).

Publisher's Note Springer Nature remains neutral with regard to jurisdictional claims in published maps and institutional affiliations.

\section{REFERENCES}

[1] Alabau-Boussouira F., Convexity and weighted integral inequalities for energy decay rates of nonlinear dissipative hyperbolic systems, Appl. Math. Opt. 51(1), 61-105 (2005).

[2] Alabau-Boussouira F., Piecewise multiplier method and nonlinear integral inequalities for Petrowsky equation with nonlinear dissipation, J. Evol. Equ. 6, 95-112 (2006).

The original article can be found online at https://doi.org/10.1007/s00028-017-0392-4. 


\section{Mohammad M. Al-Gharabli}

The Preparatory Year Program, Department of Mathematics King Fahd University of Petroleum and Minerals Dhahran 31261

Saudi Arabia

E-mail:mahfouz@kfupm.edu.sa

Salim A. Messaoudi

Department of Mathematics and Statistics

King Fahd University of Petroleum and Minerals

Dhahran 31261

Saudi Arabia

E-mail: messaoud@kfupm.edu.sa 